Originalveröffentlichung in: Bauer, Hermann ; Dittmann, Lorenz ; Piel, Friedrich (Hrsgg.): Probleme der Kunstwissenschaft, Zweiter Band: Wandlungen des Paradiesischen und Utopischen; Studien zum Bild eines Ideals, Berlin 1966, S. 279-300

\title{
Die Idee des Gottesreiches und die Philosophie der Kunst
}

\author{
von
}

\section{LORENZ DITTMANN}

I.

Das Hauptmotiv in Kants "Kritik der Urteilskraft" ist der eigentümliche Zusammenhang von reiner Subjektivität und Allgemeingültigkeit im Geschmacksurteil. Dies ist das Entscheidende, wenn Schönheit und Kunst durch das Medium der Beurteilung gefaßt werden sollen.

Das Geschmacksurteil ist „ästhetisch“, das heißt, es ist ein Urteil, „dessen Bestimmungsgrund nicht anders als subjektiv sein kann ... Hier wird die Vorstellung gänzlich auf das Subjekt, und zwar auf das Lebensgefühl desselben, unter dem Namen des Gefühls der Lust oder der Unlust, bezogen ..." (Kr. d. U. $₫ 1, V, 279^{1}$.) Deshalb sind alle Geschmacksurteile, der logischen Quantität nach, einzelne Urteile. „Denn weil ich den Gegenstand unmittelbar an mein Gefühl der Lust und Unlust halten muß, und doch nicht durch Begriffe, so kann es nicht die Quantität eines objektiv-gemeingültigen Urteils haben." $(\$ 8,293$.

Obwohl das Geschmacksurteil subjektiv und einzeln ist, erhebt es Anspruch auf allgemeine Geltung. „Man will das Objekt seinen eigenen Augen unterwerfen, gleich als ob sein Wohlgefallen von der Empfindung abhinge; und dennoch, wenn man den Gegenstand alsdann schön nennt, glaubt man eine allgemeine Stimme für sich zu haben, und macht Anspruch auf den Beitritt von jedermann, da hingegen jede Privatempfindung nur für ihn allein und sein Wohlgefallen entscheiden würde.“ $(\$ 8,294$. $)$ „Zuerst muß man sich davon völlig überzeugen: daß man durch das Geschmacksurteil (über das Schöne) das Wohlgefallen an einem Gegenstande jedermann ansinne, ohne sich doch auf einem Begriffe zu gründen (denn da wäre es das Gute); und daß dieser Anspruch auf Allgemeingültigkeit so wesentlich zu einem Urteil gehöre, wodurch wir etwas für schön erklären, daß, ohne dieselbe dabei zu denken, es niemand in den Gedanken kommen würde, diesen Ausdruck zu gebrauchen, sondern alles, was ohne Begriff gefällt, zum Angenehmen gezählt werden würde, in Ansehung dessen man jeglichem seinen Kopf für sich haben läßt, und keiner dem andern Einstimmung

${ }^{1}$ Die Werke Kants werden zitiert nach der Studienausgabe in sechs Bänden, herausgegeben von Wilhelm Weischedel, Darmstadt 1956-1964. 
zu seinem Geschmacksurteile zumutet, welches doch im Geschmacksurteile über Schönheit jederzeit geschieht." ( $(\$ 8,291$.)

Möglich ist dies, weil sich, erstens, das Geschmacksurteil auf keine „besondere Sinnesart" bezieht, vielmehr auf "dasjenige Subjektive, welches man in allen Menschen ... voraussetzen kann ... (\$38,384/385), nämlich das Gefühl der Lust, das resultiert aus dem "freien Spiel“ von Einbildungskraft und Verstand $(\$ 9,296)$ in der Erfahrung des Schönen, auf die harmonische, proportionierte Beschäftigung dieser beiden Erkenntnisvermögen in ihrer Freiheit. ( $(39,388$.$) Zweitens: weil das Wohlgefallen, welches das Geschmacksurteil$ bestimmt, ohne alles Interesse ist. "Interesse wird das Wohlgefallen genannt, was wir mit der Vorstellung der Existenz eines Gegenstandes verbinden ... Nun will man aber, wenn die Frage ist, ob etwas schön sei, nicht wissen, ob uns, oder irgend jemand, an der Existenz der Sache irgend etwas gelegen sei, oder auch nur gelegen sein könne; sondern, wie wir sie in der bloßen Betrachtung (Anschauung oder Reflexion) beurteilen." $(\$ 2,280$.) Das Geschmacksurteil ist „bloß kontemplativ" und gerade dadurch verweist es über die isolierte Subjektivität hinaus. Es beläßt den Gegenstand in seiner Freiheit, seiner Selbständigkeit. Einzig und allein das Wohlgefallen des Geschmacks am Schönen ist ein uninteressiertes und freies Wohlgefallen. Es bezieht sich auf Gunst. "Gunst ist das einzig freie Wohlgefallen. Ein Gegenstand der Neigung “ (und der Begierde) „und einer, welcher durch ein Vernunftgesetz uns zum Begehren auferlegt wird, lassen uns keine Freiheit, uns selbst irgend woraus einen Gegenstand der Lust zu machen. Alles Interesse setzt Bedürfnis voraus, oder bringt eines hervor; und, als Bestimmungsgrund des Beifalls, läßt es das Urteil über den Gegenstand nicht mehr frei sein." $(\$ 5,287$.$) Gerade als inter-$ esseloses Wohlgefallen erhebt es Anspruch auf Allgemeingültigkeit. „Denn das, wovon jemand sich bewußt ist, daß das Wohlgefallen an demselben bei ihm selbst ohne alles Interesse sei, das kann derselbe nicht anders als so beurteilen, daß es einen Grund des Wohlgefallens für jedermann enthalten müsse. Denn da es sich nicht auf irgend eine Neigung des Subjekts (noch auf irgend ein anderes überlegtes Interesse) gründet, sondern da der Urteilende sich in Ansehung des Wohlgefallens, welches er dem Gegenstande widmet, völlig frei fühlt: so kann er keine Privatbedingungen als Gründe des Wohlgefallens auffinden, an die sich sein Subjekt allein hinge, und muß es daher als in demjenigen begründet ansehen, was er auch bei jedem andern voraussetzen kann; folglich muß er glauben Grund zu haben, jedermann ein ähnliches Wohlgefallen zuzumuten." $(\$ 6,288$.)

Der Geschmack, das Vermögen der Beurteilung des Schönen aufgrund der Ubereinstimmung der Erkenntnisvermögen zur Lust, ist somit das Organ eines Gemeinsinnes. Nur unter Voraussetzung eines Gemeinsinnes kann das Geschmacksurteil gefällt werden. $(\$ 20,321$.) „Unter dem sensus communis aber 
muß man die Idee eines gemeinschaftlichen Sinnes, d. i. eines Beurteilungsvermögens verstehen, welches in seiner Reflexion auf die Vorstellungsart jedes andern in Gedanken (a priori) Rücksicht nimmt, um gleichsam an die gesamte Menschenvernunft sein Urteil zu halten, und dadurch der Illusion zu entgehen, die aus subjektiven Privatbedingungen, welche leicht für objektiv gehalten werden könnten, auf das Urteil nachteiligen Einfluß haben würde. Dieses geschieht nun dadurch, daß man sein Urteil an anderer, nicht sowohl wirkliche, als vielmehr bloß mögliche Urteile hält, und sich in die Stelle jedes andern versetzt, indem man bloß von den Beschränkungen, die unserer eigenen Beurteilung zufälliger Weise anhängen, abstrahiert..." $(\mathbb{S} 40,389$.) Geschmack als Gemeinsinn befördert damit die Humanität: „Empirisch interessiert das Schöne nur in der Gesellschaft; und, wenn man den Trieb zur Gesellschaft als dem Menschen natürlich, die Tauglichkeit aber und den Hang dazu, d. i. die Geselligkeit, zur Erfordernis des Menschen, als für die Gesellschaft bestimmten Geschöpfs, also als zur Humanität gehörige Eigenschaft einräumt: so kann es nicht fehlen, daß man nicht auch den Geschmack als ein Beurteilungsvermögen alles dessen, wodurch man sogar sein Gefübl jedem andern mitteilen kann, mithin als Beförderungsmittel dessen, was eines jeden natürliche Neigung verlangt, ansehen sollte." (\$ $\$ 41,393$.)

Die Lust, die aus der freien Übereinstimmung der Erkenntnisvermögen entspringt und das Kriterium des Geschmacksurteiles bildet, verweist noch auf Tieferes. Dies, in der „Kritik der Urteilskraft" nur angedeutet, läßt sich in Reflexionen Kants deutlicher fassen. Walter Biemel schreibt darüber: „Ist die Lust aber nur Lust als Bekundung der vollzogenen Zusammenstimmung, so muß jeweils aufgewiesen werden, was zusammenstimmt und in welcher Weise die Zusammenstimmung vollzogen wird. Unter den Reflexionen Kants finden wir eine, die die Lust vom Stimmen her kennzeichnet: 'Was mit mir selbst zusammenstimmt, in so fern ich als ein individuum der sinnenwelt mich betrachte, ist angenehm; was mit mir, als durch das ganze der Sinnenwelt bestimmt, harmonirt, ist schön; was mit mir als einem Glied der intellectualen Welt zusammenstimmt, ist gut ...'(Reflexion 712) ... Aufschlußreich ist besonders die Reflexion 1820 a: 'Die Schönen Dinge zeigen an, daß der Mensch in die Welt passe und selbst seine Anschauung der Dinge mit den Gesetzen seiner Anschauung stimme.' Diese letzte Kennzeichnung der Stimmung ist eigentlich die umfassendste. Sie leuchtet nur einen Augenblick auf, denn in der 'Kritik der Urteilskraft' beschränkt Kant die Stimmung auf das Zusammenstimmen der Vermögen des Subjekts. Aber dieses Zusammenstimmen ist von etwas ausgelöst, das mit uns in Einklang sein muß, denn auch in diesem Zusammenstimmen ist so etwas wie eine 'Anteilnahme' vorausgesetzt, so daß die Stimmung zugleich einen subjektiven und objektiven Charakter offenbart. Wenn Kant sagt: 'Schönheit ist die Beschaffenheit eines Gegenstandes oder Erkenntnisses, 
wodurch die Erkenntnisvermögen in harmonische Stimmung gesetzt werden' (Reflexion 1855) - so ist die harmonische Stimmung des Subjekts nur möglich, weil zugleich eine Harmonie zwischen dem Gegebenen und dem Subjekt herrscht, was dann das 'in die Welt passen des Menschen' genannt werden kann. " 2

In der "Kritik der Urteilskraft“ wird dies Stimmende angesprochen als das „Übersinnliche“. Es ist sowohl das „Übersinnliche in uns“, „das übersinnliche Substrat der Menschheit" $(\$ 57,445)$ wie "das intelligible Substrat der Natur außer uns". (Anmerkung II zu $\int 57,451$.) Auf dies Übersinnliche verweisen die Antinomien der Erkenntnisvermögen. „Ein bestimmtes objektives Prinzip des Geschmacks, wonach die Urteile desselben geleitet, geprüft und bewiesen werden könnten, zu geben, ist schlechterdings unmöglich; denn es wäre alsdenn kein Geschmacksurteil. Das subjektive Prinzip, nämlich die unbestimmte Idee des Übersinnlichen in uns, kann nur als der einzige Schlüssel der Enträtselung dieses uns selbst seinen Quellen nach verborgenen Vermögens angezeigt, aber durch nichts weiter begreiflich gemacht werden." $(\$ 57,446$. Wie in der "Kritik der reinen Vernunf “ und der "Kritik der praktischen Vernunft" nötigt auch in der „Kritik der Urteilskraft" die Konsequenz des Gedankenganges „über das Sinnliche hinaus zu sehen, und im Ubersinnlichen den Vereinigungspunkt aller unserer Vermögen a priori zu suchen: weil kein anderer Ausweg übrig bleibt, die Vernunft mit sich selbst einstimmig zu machen." $(\$ 57,447$.$) Und in spezieller Analogie zur praktischen Vernunft kann nun$ geurteilt werden: „Das Schöne ist das Symbol des Sittlichguten; und auch nur in dieser Rücksicht (einer Beziehung, die jedermann natürlich ist, und die auch jedermann andern als Pflicht zumutet) gefällt es, mit einem Anspruche auf jedes andern Beistimmung, wobei sich das Gemüt zugleich einer gewissen Veredlung und Erhebung über die bloße Empfänglichkeit einer Lust durch Sinneneindrücke bewußt ist, und anderer Wert auch nach einer ähnlichen Maxime ihrer Urteilskraft schätzet. Das ist das Intelligibele, worauf . . . der Geschmack hinaussieht, wozu nämlich selbst unsere oberen Erkenntnisvermögen zusammenstimmen ...“ $(\$ 59,461$.

Das ästhetische Geschmacksurteil ist von „subjektiver Allgemeingültigkeit“" $(\mathbb{8} 8,293$.$) Es „postuliert nicht jedermanns Einstimmung“, sondern „sinnet nur$ jedermann diese Einstimmung an.“ $(\$ 8,294$.$) "Man wirbt um jedes andern$ Beistimmung, weil man dazu einen Grund hat, der allen gemein ist" $(\$ 19,320)$ und läßt damit jedes anderen Freiheit unangetastet.

Das Geschmacksurteil gründet in der Lust. Deren "befreiender Charakter" zeigt sich zunächst einfach als Freiheit des Spiels - als freies Zusammenspiel

2 Walter Biemel: Die Bedeutung von Kants Begründung der Asthetik für die Philosophie der Kunst. Kantstudien. Ergänzungshefte, 77. Köln 1959, 126/127. 
der Vermögen. "Aber das eigentlich befreiende Moment des Kunstwerks liegt tiefer", liegt darin, daß es dem Subjekt zu seinem Bei-sich-selbst-Sein verhilft. „Das Subjekt ist ja nur dann bei sich selbst, wenn es seine Einheit verwirklicht. “3 Zugleich erfährt es hierin, daß es „in die Welt paßt“.

Als interesseloses wendet sich das Geschmacksurteil in „Gunst“, in „freiem Wohlgefallen" dem Gegenstande zu und entschränkt gleichzeitig das Subjekt von seinen zufälligen Privatbedingungen, erhebt es im "Gemeinsinn" zur "gesamten Menschenvernunft" und stimmt es zur Humanität. In alledem verweist es auf das Höchste, das „Übersinnliche“, das „intelligible Substrat der Natur außer uns und in uns".

Hier leuchtet in der kritischen Analyse des ästhetischen Geschmacksurteils, worin Kant den einzig zureichenden Ansatzpunkt zur Erfassung des Schönen und der Kunst zu haben glaubt, die Idee eines Reiches auf, in dem der Mensch bei sich selbst ist, versöhnt in einer freien Gemeinschaft, im Einklang mit der Natur und hingeordnet auf das "Ubersinnliche“, das alles trägt und alles eint, im letzten die Idee „Gott“.

In Kants „Kritik der Urteilskraft" wird Kunst enstmalig in ihrer Eigenständigkeit und Autonomie erfaßt und in genauen Analysen von Natur, Erkenntnis und Sittlichkeit abgehoben.

Voraussetzung hierfür und zugleich Folge ist der neue Anspruch der Philosophie auf systematische Totalität. Nur aus dem Ganzen des Seienden kann der Ort der Kunst sachgerecht bestimmt werden. „Es ist in der Tat die Doppelbestimmung und die Doppelleistung der Kantischen Philosophie, daß sie vermittelst einer neuen kritischen Isolierung der Gebiete und Probleme eine neue Synthese zwischen ihnen erschaft und begründet. " 4

Gefordert wird die "Architektonik der reinen Vernunft". Architektonik ist die „Kunst der Systeme“ und ein System „die Einheit der mannigfaltigen Erkenntnisse unter einer Idee. Diese ist der Vernunftbegriff von der Form eines Ganzen, so fern durch denselben der Umfang des Mannigfaltigen so wohl, als die Stelle der Teile untereinander, a priori bestimmt wird ... Die Einheit des Zwecks, worauf sich alle Teile und in der Idee desselben auch untereinander beziehen, macht, daß ein jeder Teil bei der Kenntnis der übrigen vermißt werden kann, und keine zufällige Hinzusetzung, oder unbestimmte Größe der Vollkommenheit, die nicht ihre a priori bestimmte Grenzen habe, stattfindet. Das Ganze ist also gegliedert (articulatio) und nicht gehäuft (coacer-

Biemel, 130.

${ }^{4}$ Ernst Cassirer: Freiheit und Form. Studien zur deutschen Geistesgeschichte. Drittes Kapitel: Die Freiheitsidee im System des kritischen Idealismus. Darmstadt 1961 ${ }^{3}, 141$. 
vatio); es kann zwar innerlich (per intus susceptionem), aber nicht äußerlich (per appositionem) wachsen, wie ein tierischer Körper, dessen Wachstum kein Glied hinzusetzt, sondern, ohne Veränderung der Proportion, ein jedes zu seinen Zwecken stärker und tüchtiger macht “. (Kritik der reinen Vernunft. Der transzendentalen Methodenlehre drittes Hauptstück: Die Architektonik der reinen Vernunft. II, 695/696.)

Aus solch kritisch-systematischer Totalität heraus wird es gelingen, daß „dasjenige, was viele Jahrhunderte nicht leisten konnten, noch vor Ablauf des gegenwärtigen erreicht werden möge: nämlich, die menschliche Vernunft in dem, was ihre Wißbegierde jederzeit, bisher aber vergeblich, beschäftigt hat, zur völligen Befriedigung zu bringen". (Kritik der reinen Vernunft. Der transzendentalen Methodenlehre viertes Hauptstück: Die Geschichte der reinen Vernunft. II, 712.)

So erweist sich das System des kritischen Idealismus als ein System der Hoffnung. "Alles Interesse meiner Vernunft (das spekulative sowohl, als das praktische) vereinigt sich in folgenden drei Fragen: 1. Was kann ich wissen? 2. Was soll ich tun? 3. Was darf ich hoffen? Die erste Frage ist bloß spekulativ ... Die zweite Frage ist bloß praktisch ... Die dritte Frage, nämlich: wenn ich nun tue, was ich soll, was darf ich alsdann hoffen? ist praktisch und theoretisch zugleich, so, daß das Praktische nur als ein Leitfaden zu Beantwortung der theoretischen, und, wenn diese hoch geht, spekulativen Frage führet. Denn alles Hoffen geht auf Glückseligkeit, und ist in Absicht auf das Praktische und das Sittengesetz eben dasselbe, was das Wissen und das Naturgesetz in Ansehung der theoretischen Erkenntnis der Dinge ist. " (Kritik der reinen Vernunft. Des Kanons der reinen Vernunft zweiter Abschnitt: Von dem Ideal des höchsten Guts, als einem Bestimmungsgrunde des letzten Zwecks der reinen Vernunft. II, 677.)

Das theoretische Wissen empfindet die Lust, daß seine Gesetze mit dem Mannigfaltigen der Natur zusammenstimmen. „Die gedachte Übereinstimmung der Natur in der Mannigfaltigkeit ihrer besonderen Gesetze zu unserem Bedürfnisse, Allgemeinheit der Prinzipien für sie aufzufinden, muß, nach aller unserer Einsicht, als zufällig beurteilt werden, gleichwohl aber doch, für unser Verstandesbedürfnis, als unentbehrlich, mithin als Zweckmäßigkeit, wodurch die Natur mit unserer, aber nur auf Erkenntnis gerichteten, Absicht übereinstimmt." - „Diese Voraussetzung der Urteilskraft ist gleichwohl darüber so unbestimmt: wie weit jene idealische Zweckmäßigkeit der Natur für unser Erkenntnisvermögen ausgedehnt werden solle, daß, wenn man uns sagt, eine tiefere oder ausgebreitetere Kenntnis der Natur durch Beobachtung müsse zuletzt auf eine Mannigfaltigkeit von Gesetzen stoßen, die kein menschlicher Verstand auf ein Prinzip zurückführen kann, wir es auch zufrieden sind, ob 
wir es gleich lieber hören, wenn andere uns Hoffnung geben: daß, je mehr wir die Natur im Inneren kennen würden, oder mit äußeren uns für jetzt unbekannten Gliedern vergleichen könnten, wir sie in ihren Prinzipien um desto einfacher, und, bei der scheinbaren Heterogeneität ihrer empirischen Gesetze einhelliger finden würden, je weiter unsere Erfahrung fortschritte." (Kritik der Urteilskraft, Einleitung. VI. Von der Verbindung des Gefühls der Lust mit dem Begriffe der Zweckmäßigkeit der Natur. V, 260, 262.)

Dieser theoretischen Verstandeshoffnung tritt zur Seite die praktische Vernunfthoffnung. "Ich nenne die Welt, so fern sie allen sittlichen Gesetzen gemäß wäre ... eine moralische Welt." Diese ist "eine bloße, aber doch praktische Idee, die wirklich ihren Einfluß auf die Sinnenwelt haben kann und soll, um sie dieser Idee so viel als möglich gemäß zu machen. Die Idee einer moralischen Welt hat daher objektive Realität, nicht als wenn sie auf einen Gegenstand einer intelligibelen Anschauung ginge (dergleichen wir uns gar nicht denken können), sondern auf die Sinnenwelt, aber als einen Gegenstand der reinen Vernunft in ihrem praktischen Gebrauche, und ein corpus mysticum der vernünftigen Wesen in ihr, so fern deren freie Willkür unter moralischen Gesetzen sowohl mit sich selbst, als mit jedes anderen Freiheit durchgängige systematische Einheit an sich hat". Auf die Frage nun: „wie, wenn ich mich nun so verhalte, daß ich der Glückseligkeit nicht unwürdig sei, darf ich auch hoffen, ihrer dadurch teilhaftig werden zu können?" ist zu antworten: „daß eben sowohl, als die moralischen Prinzipien nach der Vernunft in ihrem praktischen Gebrauche notwendig sind, eben so notwendig sei es auch nach der Vernunf, in ihrem theoretischen Gebrauch anzunehmen, daß jedermann die Glückseligkeit in demselben Maße zu hoffen Ursache habe, als er sich derselben in seinem Verhalten würdig gemacht hat, und daß also das System der Sittlichkeit mit dem der Glückseligkeit unzertrennlich, aber nur in der Idee der reinen Vernunft verbunden sei.“ „Aber dieses System der sich selbst lohnenden Moralität ist nur eine Idee, deren Ausführung auf der Bedingung beruht, daß jedermann tue, was er soll, d. i. alle Handlungen vernünftiger Wesen so geschehen, als ob sie aus einem obersten Willen, der alle Privatwillkür in sich, oder unter sich befaßt, entsprängen... (Kritik der reinen Vernunft. Des Kanons der reinen Vernunft zweiter Abschnitt: Von dem Ideal des höchsten Guts, als einem Bestimmungsgrunde des letzten Zwecks der reinen Vernunft. II, 679, 680.)

"Das Prinzip der Zweckmäßigkeit der Natur und die Idee des Systems der sich selbst lohnenden Moralität bilden die beiden Systeme der Hoffnung. "s Das System der Urteilskraft jedoch verbindet die beiden Systeme des Verstandes und der Vernunft, des Wissens und des Willens. „Die Geschmacks-

${ }^{5}$ Helmuth Plessner: Die Einheit der Sinne. Grundlinien einer Aesthesiologie des Geistes. Bonn 1923, 389, 390. 
kritik... eröffnet, wenn man sie in transzendentaler Absicht behandelt, dadurch, daß sie eine Lücke im System unserer Erkenntnisvermögen ausfüllt, eine auffallende und wie mich dünkt viel verheißende Aussicht in ein vollständiges System aller Gemütskräfte, so fern sie in ihrer Bestimmung nicht allein aufs Sinnliche, sondern auch aufs Ubersinnliche bezogen sind ..." (Einleitung in die Kritik der Urteilskraft. Erste Fassung. XI. Enzyklopädische Introduktion der Kritik der Urteilskraft in das System der Kritik der reinen Vernunft. V, 224.)

Der „Kritik der Urteilskraft, als einem Verbindungsmittel der zwei Teile der Philosophie zu einem Ganzen" (K. d. U., Einleitung. V, 248) kommt in solcher Einheitsstiftung mithin vorzüglich der Charakter eines Systems der Hoffnung und seiner Verweisung auf Glückseligkeit zu: und damit dem Schönen und der Kunst. Dies ist von Kant nicht ausgesprochen worden, ergibt sich aber aus der Schlüssigkeit des Gedankenzusammenhanges und wurde zum treibenden Impuls der kunstphilosophischen Reflexion bei Schiller und Schelling.

An einer Stelle nimmt die "Kritik der Urteilskraft" ausdrücklich Bezug auf die Leitbegriffe des philosophischen Chiliasmus: Freiheit, Selbsttätigkeit, Mündigkeit, Erweiterung zur Menschheit. Es ist die „Episode“ zur Erläuterung der Grundsätze der Geschmackskritik. Hier heißt es ( $\$ 40$, V, 390/391): „Folgende Maximen des gemeinen Menschenverstandes gehören zwar nicht hierher, als Teile der Geschmackskritik, können aker doch zur Erläuterung ihrer Grundsätze dienen. Es sind folgende: 1. Selbstdenken; 2. An der Stelle jedes andern denken; 3. Jederzeit mit sich selbst einstimmig denken. Die erste ist die Maxime der vorurteilsfreien, die zweite der erweiterten, die dritte der konsequenten Denkungsart. Die erste ist die Maxime einer niemals passiven Vernunft. Der Hang zur letztern, mithin zur Heteronomie der Vernunft, heißt das Vorurteil; und das größte unter allen ist, sich die Naturregeln, welche der Verstand ihr durch ihr eigenes wesentliches Gesetz zum Grunde legt, als nicht unterworfen vorzustellen: d. i. der Aberglaube. Befreiung vom Aberglauben heißt Aufklärung; weil, obschon diese Benennung auch der Befreiung von Vorurteilen überhaupt zukommt, jener doch vorzugsweise (in sensu eminenti) ein Vorurteil genannt zu werden verdient, indem die Blindheit, worin der Aberglaube versetzt, ja sie wohl gar als Obliegenheit fordert, das Bedürfnis, von andern geleitet zu werden, mithin den Zustand einer passiven Vernunft vorzüglich kenntlich macht. Was die zweite Maxime der Denkungsart betriff, so sind wir sonst wohl gewohnt, denjenigen eingeschränkt (borniert, das Gegenteil von erweitert) zu nennen, dessen Talente zu keinem großen Gebrauche (vornehmlich dem intensiven) zulangen. Allein hier ist nicht die Rede vom Vermögen des Erkenntnisses, sondern von der Denkungsart, einen zweckmäßigen Gebrauch davon zu machen; welche, so klein auch der Umfang und der Grad 
sei, wohin die Naturgabe des Menschen reicht, dennoch einen Mann von erweiterter Denkungsart anzeigt, wenn er sich über die subjektiven Privatbedingungen des Urteils, wozwischen so viele andere wie eingeklammert sind, wegsetzen, und aus einem allgemeinen Standpunkte (den er dadurch nur bestimmen kann, daß er sich in den Standpunkt anderer versetzt) über sein eigenes Urteil reflektiert. Die dritte Maxime, nämlich die der konsequenten Denkungsart, ist am schwersten zu erreichen, und kann auch nur durch die Verbindung beider ersten, und nach einer zur Fertigkeit gewordenen öfteren Befolgung derselben, erreicht werden ....

Bezeichnend sind auch die Beispiele für „ästhetische Attribute“ der Gegenstände, die eine „ästhetische Idee“ vermitteln und so „der Einbildungskraft einen Schwung geben, mehr dabei, obzwar auf unentwickelte Art zu denken, als sich in einem Begriffe, mithin in einem bestimmten Sprachausdrucke, zusammenfassen läßt“" „Wenn der Große König sich in einem seiner Gedichte so ausdrückt: ,Laßt uns aus dem Leben ohne Murren weichen und ohne etwas zu bedauern, indem wir die Welt noch alsdann mit Wohltaten überhäuft zurücklassen. So verbreitet die Sonne, nachdem sie ihren Tageslauf vollendet hat, noch ein mildes Licht am Himmel; und die letzten Strahlen, die sie in die Lüfte schickt, sind ihre letzten Seufzer für das Wohl der Welt' : so belebt er seine Vernunftidee, von weltbürgerlicher Gesinnung noch am Ende des Lebens, durch ein Attribut, welches die Einbildungskraft (in der Erinnerung an alle Annehmlichkeiten eines vollbrachten schönen Sommertages, die uns ein heiterer Abend ins Gemüt ruft) jener Vorstellung beigesellt, und welches eine Menge von Empfindungen und Nebenvorstellungen rege macht, für die sich kein Ausdruck findet. Andererseits kann sogar ein intellektueller Begriff umgekehrt zum Attribut einer Vorstellung der Sinne dienen, und so diese letztern durch die Idee des Ubersinnlichen beleben; aber nur, indem das ästhetische, was zum Bewußtsein des letztern subjektiv anhänglich ist, hiezu gebraucht wird. So sagt z. B. ein gewisser Dichter in der Beschreibung eines schönen Morgens: ,Die Sonne quoll hervor, wie Ruh aus Tugend quillt'. Das Bewußtsein der Tugend, wenn man sich auch nur in Gedanken in die Stelle eines Tugendhaften versetzt, verbreitet im Gemüte eine Menge erhabener und beruhigender Gefühle, und eine grenzenlose Aussicht in eine frohe Zukunft, die kein Ausdruck, welcher einem bestimmten Begriffe angemessen ist, völlig erreicht." ( $(49, \mathrm{~V}, 416 / 417$.

In jener „Episode“ klingen die Thesen der „Beantwortung der Frage: Was ist Aufklärung?" nach: „Aufklärung ist der Ausgang des Menschen aus seiner selbst verschuldeten Unmündigkeit. Unmündigkeit ist das Unvermögen, sich seines Verstandes ohne Leitung eines anderen zu bedienen. Selbstverschuldet ist diese Unmündigkeit, wenn die Ursache derselben nicht am Mangel des Verstandes, sondern der Entschließung und des Mutes liegt, sich seiner ohne Leitung eines andern zu bedienen. Sapere aude! Habe Mut, dich deines eigenen Verstandes zu bedienen! ist also der Wahlspruch der Aufklärung." (VI, 53.)

Solchem Aufruf zum Selbstdenken, zur Selbsttätigkeit und Selbstverantwortung („es ist so bequem, unmündig zu sein“) liegt die Überzeugung der Vollendungsmöglichkeit des Menschengeschlechtes zugrunde. „Man kann die Geschichte der Menschengattung im großen als die Vollziehung eines verborgenen Plans der Natur ansehen, um eine innerlich - und, zu diesem Zwecke, auch äußerlich - vollkommene Staatsverfassung zu Stande zu bringen als den einzigen Zustand, in welchem sie alle ihre Anlagen in der Menschheit völlig ent- 
wickeln kann. ... Man sieht: die Philosophie könne auch ihren Chiliasmus haben; aber einen solchen, zu dessen Herbeiführung ihre Idee, obgleich nur sehr von weitem, selbst beförderlich werden kann, der also nichts weniger als schwärmerisch ist..." (Idee zu einer allgemeinen Geschichte in weltbürgerlicher Absicht. VI, 45.)

Autonomie des Willens ist oberstes Prinzip der Sittlichkeit. Dieses Prinzip besagt, „daß der Wille durch seine Maxime sich selbst zugleich als allgemein gesetzgebend betrachten könne" oder als kategorischer Imperativ: „handle nach der Maxime, die sich selbst zugleich zum allgemeinen Gesetz machen kann." (Grundlegung zur Metaphysik der Sitten. Zweiter Abschnitt. IV, 67, 70.)

Von hier aus ist die Idee einer "moralischen Welt", eines „ethischen gemeinen Wesens" „unter Gründung eines Reichs Gottes auf Erden" entworfen. (Die Religion innerhalb der Grenzen der bloßen Vernunft. Drittes Stück. IV, 751 ff.) „Ein moralisches Volk Gottes zu stiften, ist (jedoch) ein Werk, dessen Ausführung nicht von Menschen, sondern nur von Gott selbst erwartet werden kann. Deswegen ist aber doch dem Menschen nicht erlaubt, in Ansehung dieses Geschäftes untätig zu sein, und die Vorsehung walten zu lassen, als ob ein jeder nur seiner moralischen Privatangelegenheit nachgehen, das Ganze der Angelegenheit des menschlichen Geschlechts aber (seiner moralischen Bestimmung nach) einer höhern Weisheit überlassen dürfe. Er muß vielmehr so verfahren, als ob alles auf ihn ankomme, und nur unter dieser Bedingung darf er hoffen, daß höhere Weisheit seiner wohlgemeinten Bemühung die Vollendung werde angedeihen lassen." (Ebenda, IV, 760.)

Weil die Gründung eines Reiches Gottes auf Erden von der moralischen Anstrengung und Selbstverantwortung der mündigen Menschen abhängig ist - Kant stuft den paradiesischen Urzustand niedrig ein ${ }^{6}$ - deshalb ist sie eine ständige, nur in kontinuierlichem Fortschreiten und in Annäherung zu verwirklichende Aufgabe der Zukunft. Die „erhabene Idee eines ethischen gemeinen Wesens“ ist nie völlig erreichbar. Denn: „Wie kann man erwarten, daß

${ }^{6}$ „.... würden in einem arkadischen Schäferleben, bei vollkommener Eintracht, Genügsamkeit und Wechselliebe, alle Talente auf ewig in ihren Keimen verborgen bleiben: die Menschen, gutartig wie die Schafe die sie weiden, würden ihrem Dasein kaum einen größeren Wert verschaffen, als dieses ihr Hausvieh hat..." (Idee zu einer allgemeinen Geschichte in weltbürgerlicher Absicht. Vierter Satz. VI, 38.) - „Aus dieser Darstellung der ersten Menschengeschichte ergibt sich: daß der Ausgang des Menschen aus dem, ihm durch die Vernunf, als erster Aufenthalt seiner Gattung vorgestellten, Paradiese nicht anders, als der Obergang aus der Rohigkeit eines bloß tierischen Geschöpfes in die Menschheit, aus dem Gängelwagen des Instinkts zur Leitung der Vernunft, mit einem Worte: aus der Vormundschaft der Natur in den Stand der Freiheit gewesen sei. Ob der Mensch durch diese Veränderung gewonnen, oder verloren habe, kann nun nicht mehr die Frage sein, wenn man auf die Bestimmung seiner Gattung sieht, die in nichts als im Fortschreiten zur Vollkommenheit besteht..." (Mutmaßlicher Anfang der Menschengeschichte. Anmerkung. VI, 92.) 
aus so krummen Holze (als woraus der Mensch gemacht ist), etwas völlig Gerades gezimmert werde? “ (Ebenda, IV, 760; vgl. VI, 41.)

Autonomie, das heißt: Selbstdenken und Selbsthandeln, ist die unnachläßliche Voraussetzung dieses Reiches Gottes, selbst für die Beurteilung, was ein Reich Gottes, wer Gott sei. So heißt es in einer kühnen Formulierung: „Es klingt zwar bedenklich, ist aber keineswegs verwerflich, zu sagen: daß ein jeder Mensch sich einen Gott mache, ja nach moralischen Begriffen ... sich einen solchen selbst machen müsse, um an ihm den, der ibn gemacht hat, zu verehren. Denn auf welcherlei Art auch ein Wesen als Gott von einem anderen bekannt gemacht und beschrieben worden, ja ihm ein solches auch (wenn das möglich ist) selbst erscheinen möchte, so muß er diese Vorstellung doch allererst mit seinem Ideal zusammen halten, um zu urteilen, ob er befugt sei, es für eine Gottheit zu halten und zu verehren. ${ }^{\text {"7 }}$ (Ebenda, IV, 839/840.)

Autonomie ist Grundprinzip der Kantischen Philosophie und bestimmt auch die „Kritik der Urteilskraft“. Ganz ähnlich wird vom Geschmack gesagt, „daß das höchste Muster, das Urbild des Geschmacks, eine bloße Idee sei, die jeder in sich selbst hervorbringen muß, und wonach er alles, was Objekt des Geschmacks, was Beispiel der Beurteilung durch Geschmack sei, und selbst den Geschmack von jedermann, beurteilen muß“. (K. d. U. $₫ 17$, V, 314.)

Ist aber das Reich Gottes als ethisches Gemeinwesen mündiger Menschen niemals völlig zu verwirklichen, verbleibt es hier eine erhabene, uneinholbare Idee, so ist es im Schönen und in der Kunst, wie zu erschließen war, je gegenwärtig ${ }^{8}$.

Das je gegenwärtige Reich Gottes, von dem Kunst und Schönheit Zeugnis geben, verweist, da Schönheit Symbol der Sittlichkeit ist, auf das durch den sittlichen Willen zu verwirklichende, wenn auch nur in unendlicher Annäherung erreichbare Reich Gottes auf Erden: Dies ist der Zusammenhang von Kunst und der Idee des Reiches Gottes im Kantischen System.

7 Solche Kühnheit widerspricht nicht dem Christentum. Ganz im Gegenteil, denn bei Kant sind Freiheit und Liebe ursprünglich zusammengedacht: „Wenn man nun, um es recht gut zu machen, zum Christentum noch irgend eine Auktorität (wäre es auch die göttliche) hinzutut, die Absicht derselben mag auch noch so wohlmeinend und der Zweck auch wirklich noch so gut sein: so ist doch die Liebenswürdigkeit desselben verschwunden: denn es ist ein Widerspruch, jemanden zu gebieten, daß er etwas nicht allein tue, sondern es auch gern tun solle... Es ist also die liberale Denkungsart - gleichweit entfernt vom Sklavensinn, und von Bandenlosigkeit - wovon das Christentum für seine Lehre Effekt erwartet, durch die es die Herzen der Menschen für sich zu gewinnen vermag, deren Verstand schon durch die Vorstellung des Gesetzes ihrer Pflicht erleuchtet ist. Das Gefühl der Freiheit in der Wahl des Endzwecks ist das, was ihnen die Gesetzgebung liebenswürdig macht." (Das Ende aller Dinge. VI, 188.)

8 In der Kunst gibt es keinen Fortschritt: „... weil für diese (die Genies) die Kunst irgendwo still steht, indem ihr eine Grenze gesetzt ist, über die sie nicht weiter gehen kann, die vermutlich auch schon seit lange her erreicht ist und nicht mehr erweitert werden kann;..." (K. d. U. \$ 47, V, 408.) 


\section{II.}

Schiller vertieft den kunstphilosophischen Ansatz Kants. Ihm genügt es nicht mehr, Schönheit und Kunst über die Weise der Beurteilung und aus den Vermögen des Subjekts zu erfassen. Er erkennt Schönheit und Kunst als den Bereich, der dem Menschen das Menschsein gewährt.

Schillers „Briefe über die ästhetische Erziehung des Menschen“ radikalisieren die abendländische Definition des Menschen als "animal rationale" zur Antinomie von Natur und Geist, "Zustand" und „Person", „Stofftrieb" und „Formtrieb“9. Versöhnung und Vermittlung stiftet der „ästhetische Zustand“, der „Spieltrieb“. Als Naturmensch untersteht der Mensch den Gesetzlichkeiten dieser Natur, als Vernunftwesen wird er hinaufgerissen in ein ideelles Reich, zur Selbstgesetzgebung als Person; im ästhetischen Bereich allein ist ihm die schöne Freiheit gegeben, seine Einheit nicht im Widerstand gegen eine äußere Mannigfaltigkeit behaupten zu müssen, Natur- und Geistwesen zugleich sein zu können. Hier erst ist er in sein Menschsein freigegeben. „Die Schönheit läßt den Menschen auf menschliche Weise bei sich selbst anwesend sein. "10

Kants Bestimmung des ästhetischen Zustandes als freies Spiel der Vermögen wird von Schiller aufgenommen und fortgebildet. „Der sinnliche Trieb schließt aus seinem Subjekt alle Selbsttätigkeit und Freiheit, der Formtrieb aus dem seinigen alle Abhängigkeit, alles Leiden aus. Ausschließung der Freiheit ist aber physische, Ausschließung des Leidens ist moralische Notwendigkeit. Beide Triebe nötigen also das Gemüt, jener durch Naturgesetze, dieser durch Gesetze der Vernunft. Der Spieltrieb also, als in welchem beide verbunden wirken, wird das Gemüt zugleich moralisch und physisch nötigen; er wird also, weil er alle Zufälligkeit aufhebt, auch alle Nötigung aufheben und den Menschen sowohl physisch als moralisch in Freiheit setzen." (Vierzehnter Brief, V, $613^{11}$.)

Hierbei bleibt Schiller nicht stehen. Es gilt, diesen Zustand in seinem Verhältnis zu Erkenntnis und Moralität zu fassen: „Wenn also die ästhetische Stimmung des Gemüts in einer Rücksicht als Null betrachtet werden muß, sobald man nämlich sein Augenmerk auf einzelne und bestimmte Wirkungen richtet, so ist sie in anderer Rücksicht wieder als Zustand der höchsten Realität anzusehen, insofern man dabei auf die Abwesenheit aller Schranken und auf die Summe der Kräfte achtet, die in derselben gemeinschaftlich tätig sind. Man

9 Vgl. hierzu K. H. Volkmann-Schluck: Die Kunst und der Mensch. Schillers Briefe über die ästhetische Erziehung des Menschen. Frankfurt/M. 1964 (Wissenschaft und Gegenwart. Heft 26).

10 Volkmann-Schluck, 15.

11 Zitiert nach: Friedrich Schiller. Sämtliche Werke. Hrsg. von Gerhard Fricke und Herbert G. Göpfert. Fünfter Band. Erzählungen/Theoretische Schriften. München 1960². 
kann also denjenigen ebensowenig unrecht geben, die den ästhetischen Zustand als den fruchtbarsten in Rücksicht auf Erkenntnis und Moralität erklären. Sie haben vollkommen recht; denn eine Gemütsstimmung, welche das Ganze der Menschheit in sich begreift, muß notwendig auch jede einzelne Äußerung derselben, dem Vermögen nach, in sich schließen; eine Gemütsstimmung, welche von dem Ganzen der menschlichen Natur alle Schranken entfernt, muß diese notwendig auch von jeder einzelnen Außerung derselben entfernen. Eben deswegen, weil sie keine einzelne Funktion der Menschheit ausschließend in Schutz nimmt, so ist sie einer jeden ohne Unterschied günstig, und sie begünstigt ja nur deswegen keine einzelne vorzugsweise, weil sie der Grund der Möglichkeit von allen ist. Alle andere Übungen geben dem Gemüt irgendein besonderes Geschick, aber setzen ihm dafür auch eine besondere Grenze; die ästhetische allein führt zum Unbegrenzten. Jeder andere Zustand, in den wir kommen können, weist uns auf einen vorhergehenden zurück und bedarf zu seiner Auflösung eines folgenden; nur der ästhetische ist ein Ganzes in sich selbst, da er alle Bedingungen seines Ursprungs und seiner Fortdauer in sich vereinigt. Hier allein fühlen wir uns wie aus der Zeit gerissen; und unsere Menschheit äußert sich mit einer Reinheit und Integrität, als hätte sie von der Einwirkung äußrer Kräfte noch keinen Abbruch erfahren." (Zweiundzwanzigster Brief, 636/637.)

Dieser "Zustand der Indifferenz" ist nun, innigste Vereinigung zwischen Kunst und Leben, Schönheit und dem wirklichen Reiche der Vollkommenheit herstellend, das notwendige Durchgangsstadium zu dem "vollkommensten aller Kunstwerke,... dem Bau einer wahren politischen Freiheit." (Zweiter Brief, 572.) Die Möglichkeit einer „ästhetischen Erziehung des Menschen“, der diese Briefe gewidmet sind, rechtfertigt sich einzig aus Schillers Überzeugung, „daß man, um jenes politische Problem in der Erfahrung zu lösen, durch das ästhetische den Weg nehmen muß, weil es die Schönheit ist, durch welche man zu der Freiheit wandert." (Zweiter Brief, 573.) Hier gewinnt die Erfahrung von Kunst und deren Durchleuchtung in der Theorie einen Ernst und eine Würde, vor denen spätere Bildungstheorien und Rechtfertigungen wissenschaftlicher Befassung mit Kunst den Charakter sinnfreier Wissensneugier annehmen müssen. Da die Schönheit als eine „notwendige Bedingung der Menschheit“ erkannt wurde, ist sie auch der notwendige Weg, den ein mündig gewordenes Volk einzuschlagen hat bei seinem Versuche, „seinen Naturstaat in einen sittlichen umzuformen".

Kunst, die "Tochter der Freiheit" und die Führerin zur Freiheit, ist zuerst und vor allem das Reich der Freiheit selbst: „Freibeit zu geben durch Freiheit ist das Grundgesetz dieses Reichs", des ästhetischen Staats. (Siebenundzwanzigster Brief, 667.) Der „objektive Begriff des Schönen“, um den sich Schiller bemüht, ist in der Freiheit gegründet: Schönheit ist „Freiheit in der Erschei- 
nung", "Autonomie in der Erscheinung"12. (Kallias oder über die Schönheit, Briefe an Gottfried Körner, 8. Februar 1793, V, 400.) „Die Schönheit ... betrachtet alle Dinge als Selbstzwecke und duldet schlechterdings nicht, daß eins dem andern als Mittel dient oder das Joch trägt. In der ästhetischen Welt ist jedes Naturwesen ein freier Bürger, der mit dem Edelsten gleiche Rechte hat, und nicht einmal um des Ganzen willen darf gezwungen werden, sondern zu allem schlechterdings konsentieren muß.“ (Kallias, 23. Februar 1793, 421.)

Die zweite Bestimmung der Schillerschen Schönheits- und Kunstphilosophie aber lautet: Das Wesen der Schönheit liegt in dem ästhetischen Scheine ${ }^{13}$. Als Schein ist die ästhetische Welt streng von der Wirklichkeit abgetrennt: „Da alles wirkliche Dasein von der Natur, als einer fremden Macht, aller Schein aber ursprünglich von dem Menschen, als vorstellendem Subjekte, sich herschreibt, so bedient er sich bloß seines absoluten Eigentumsrechts, wenn er den Schein von dem Wesen zurücknimmt und mit demselben nach eignen Gesetzen schaltet... Dieses menschliche Herrscherrecht übt er aus in der Kunst des Scheins, und je strenger er hier das Mein und Dein voneinander sondert, je sorgfältiger er die Gestalt von dem Wesen trennt, und je mehr Selbständigkeit er derselben zu geben weiß, desto mehr wird er nicht bloß das Reich der Schönheit erweitern, sondern selbst die Grenzen der Wahrheit bewahren; denn er kann den Schein nicht von der Wirklichkeit reinigen, ohne zugleich die Wirklichkeit von dem Schein frei zu machen. - Aber er besitzt dieses souveräne Recht schlechterdings auch nur in der Welt des Scheins, in dem wesenlosen Reich der Einbildungskraft, und nur, solange er sich im Theoretischen gewissenhaft enthält, Existenz davon auszusagen, und solang er im Praktischen darauf Verzicht tut, Existenz dadurch zu erteilen." (Sechsundzwanzigster Brief, 658.)

Als Erscheinung der Freiheit jedoch stellt die ästhetische Welt den Anspruch und die Forderung, in die Wirklichkeit einzugreifen und sie zu verwandeln. Dies ist die Aufgabe des Künstlers. Er ist „Zwar der Sohn seiner Zeit, aber schlimm für ihn, wenn er zugleich ihr Zögling oder gar ihr Günstling ist“. Ihm wird zugerufen: „Lebe mit deinem Jahrhundert, aber sei nicht sein Geschöpf; leiste deinen Zeitgenossen, aber was sie bedürfen, nicht was sie loben ... Verjage die Willkür, die Frivolität, die Rohigkeit aus ihren Vergnügungen, so wirst du sie unvermerkt auch aus ihren Handlungen, endlich aus ihren Gesinnungen verbannen. Wo du sie findest, umgib sie mit edeln, mit großen, mit geistreichen Formen, schließe sie ringsum mit den Symbolen des Vortrefflichen

12 Umfassend behandelt bei Ernst Cassirer: Freiheit und Form. Fünftes Kapitel: Schiller. Freiheitsproblem und Formproblem in der klassischen Ästhetik.

${ }^{13}$ Hierzu Volkmann-Schluck, $15 \mathrm{ff}$. und seine Analyse des ästhetischen Scheins, $22 \mathrm{ff}$.; sowie Heinz Wenzel: Das Problem des Scheins in der Asthetik. Schillers "Asthetische Briefe" Diss. Köln 1958. 
ein, bis der Schein die Wirklichkeit und die Kunst die Natur überwindet." (Neunter Brief, 593, 595, 596.)

Kunst ist Schein: das bewahrt sie davor, nur Übergang zu einem wirklichen Reiche der Vollkommenheit zu sein und in diesem aufgehoben zu werden ${ }^{14}$. Für die Wirklichkeit ist dem Menschen die "Idee seiner Menschheit" ein „Unendliches, dem er sich im Laufe der Zeit immer mehr nähern kann, aber ohne es jemals zu erreichen". Das Kunstwerk aber ist ein "Symbol seiner ausgefübrten Bestimmung", und ("weil diese nur in der Allheit der Zeit zu erreichen ist") eine "Darstellung des Unendlichen“. (Vierzehnter Brief, 612.)

Schönheit und Kunst als „Erscheinung der Freiheit" können und müssen ins wirkliche Leben eingreifen und es zu ihrer Vollkommenheit hinführen. Das Schöne, das "Symbol des in sich Vollendeten oder des Vollkommenen" (Kallias, 23. Februar 1793, 416) darf sich nicht mit seiner autonomen Sphäre, unkräftig für die Wirklichkeit, begnügen, sondern ist Vor-Schein einer wirklichen Vollendung, die von der Wirklichkeit jedoch nie einzuholen ist. „So wie die edle Kunst die edle Natur überlebte, so schreitet sie derselben auch in der Begeisterung, bildend und erweckend, voran." (Neunter Brief, 594.)

In diesem Spannungsverhältnis stehen Schönheit und Kunst und das zu verwirklichende Reich der Vollendung. Im selben Sinne verhielten sich Kunst und die Idee des Gottesreiches im Systeme Kants. Dort erst keimhaft angelegt, prägt Schiller diese Gedanken zur Grundlage seiner Theorie von Kunst und Menschentum aus.

Friedrich Kreis (Die Autonomie des Asthetischen in der neueren Philosophie. Tübingen 1922, 53/54) findet in Schillers „Briefen über die ästhetische Erziehung des Menschen“ folgenden Bruch: „In dieser Abhandlung Schillers stehen die beiden allein möglichen ästhetischen Theorien (die metaphysische und die formale), die sich aber ihrem Sinne nach ... gegenseitig ausschließen, geradezu nebeneinander; und zwar so unvermittelt, daß man zu der Annahme gezwungen wird, Schiller selbst sei die Verschiedenheit der beiden Begriffe des Schönen verborgen geblieben. Während nämlich in dem größeren Teile der Abhandlung die Harmonie von Sinnlichkeit und Vernunft das einzige Kriterium des Schönen ist, setzt nun von dem 25. Briefe an eine Betrachtungsweise ein, die vollkommen auf dem Boden der Kontemplativitätstheorie steht. Hier wird das Schöne allein bedingt durch das Moment einer ihren Gegenstand isolierenden reinen Kontemplation ... Aus der Interesselosigkeit der rein ästhetischen Betrachtung leitet nun Schiller auch die das Ästhetische näher charakterisierenden Begriffe ab, den des Scheins und den des Spiels ... Auffallend bleibt nur die Tatsache, daß Schiller den Gegensatz dieser Theorie zu aller metaphysischen Spekulation so vollkommen übersehen, oder seiner doch keine Erwähnung getan hat." Zur ersten Theorie meint Kreis: „Die harmonische Erscheinung des Ästhetischen beruht also nicht etwa auf einer Abgedrängtheit des Aisthetischen vom unmittelbaren Leben, sie ist hier nicht eine Eigenschaft des isolierten kontemplativen Gebildes, sondern ein Phänomen dieses unmittelbaren Lebens selbst. Die Harmonie von Sinnlichkeit und Vernunft ist denn in der Tat nichts anderes als das Symbol eines ethischen oder sonst eines persönlichen Wertes, nicht aber eine spezifisch ästhetische Erscheinung. Indem Schiller diese Harmonie von Sinnlichem und Ubersinnlichem selbst als ästhetisches Phänomen anspricht, hat er nach Art und Weise aller metaphysischen Asthetik einer typisch außerästhetischen

${ }^{14}$ Dies ist die Funktion der Kunst in den Utopien des Suprematismus und der Stijl-Bewegung. 
Erscheinung den Sinn des Asthetischen unterlegt. “ (51/52.) Kreis setzt hier eine Konstruktion des „Ästhetischen" (d. h. der Schönheit und der Kunst) als eines isolierten, vom Leben abgetrennten, kraftlosen Phänomens für fraglos gültig an. Diese Bedeutung des Ästhetischen ist jedoch erst für die Entwicklung von Konrad Fiedler zur modernen Kunstwissenschaft verbindlich geworden. Es gilt, im Gegenteil, die tiefere und umfassendere Bestimmung des kritischen Idealismus wieder aufzugreifen.

Bei Schiller sind Versöhnung von Sinnlichkeit und Geist, Freiheit und Kontemplation untrennbar verbunden. Hier gibt es keinen Gegensatz von Leben und Betrachtung. „Die Schönheit ist allerdings das Werk der freien Betrachtung, und wir treten mit ihr in die Welt der Ideen - aber ... ohne darin die sinnliche Welt zu verlassen." Deshalb läßt sich hier keine "Succession zwischen der Tätigkeit und dem Leiden unterscheiden, und die Reflexion zerfließt hier so vollkommen mit dem Gefühle, daß wir die Form unmittelbar zu empfinden glauben. Die Schönheit ist also zwar Gegenstand für uns, weil die Reflexion die Bedingung ist, unter der wir eine Empfindung von ihr haben; zugleich aber ist sie ein Zustand unseres Subjekts, weil das Gefühl die Bedingung ist, unter der wir eine Vorstellung von ihr haben. Sie ist also zwar Form, weil wir sie betrachten, zugleich aber ist sie Leben, weil wir sie fühlen. Mit einem Wort: sie ist zugleich unser Zustand und unsre Tat." (Fünfundzwanzigster Brief, 653/654.) Die ästhetische Betrachtung, getragen von der „hohen Freiheit des Schönen “ gewährt dem Menschen, „daß ihm die Freiheit, zu sein, was er sein soll, vollkommen zurückgegeben ist." (Einundzwanzigster Brief, 635.)

Zum Fundament historischen Verstehens von Kunst wird diese Konzeption in der Abhandlung "Uber naive und sentimentalische Dichtung“. Wie ist, im Angesicht einer vergangenen Vollkommenheit, ein Neues, Besseres möglich? Größe und Einfachheit der naiven Natur bewundernd, ist es uns aufgegeben, sie aus dem Idealen, geistgeboren, wiederzuerlangen: dies ist der Horizont einer Theorie und Geschichte der Literatur im Zeichen der Utopie.

„... Wenn du über das verlorene Glück der Natur getröstet bist, so laß ihre Vollkommenheit deinem Herzen zum Muster dienen. Trittst du heraus $\mathrm{zu}$ ihr aus deinem künstlichen Kreis, steht sie vor dir in ihrer großen Ruhe, in ihrer naiven Schönheit, in ihrer kindlichen Unschuld und Einfalt; dann verweile bei diesem Bilde, pflege dieses Gefühl, es ist deiner herrlichsten Menschheit würdig. Laß dir nicht mehr einfallen, mit ihr tauschen zu wollen, aber nimm sie in dich auf und strebe, ihren unendlichen Vorzug mit deinem eigenen unendlichen Prärogativ zu vermählen und aus beidem das Göttliche zu erzeugen." (708/709.) Denn das Ziel, „zu welchem der Mensch durch Kultur strebt, ist demjenigen, welches er durch Natur erreicht, unendlich vorzuziehen. Der eine (Weg) erhält seinen Wert durch absolute Erreichung einer endlichen, der andre erlangt ihn durch Annäherung zu einer unendlichen Größe ..." (718.)

Die wichtigste Art der sentimentalischen Dichtung, die eigentlich utopische, ist die "Idylle". Idyllen im üblichen Verstande, Hirtenidyllen, „führen uns theoretisch rückwärts, indem sie uns praktisch vorwärts führen und veredeln. Sie stellen unglücklicherweise das Ziel hinter uns, dem sie uns doch entgegenführen sollten, und können uns daher bloß das traurige Gefühl eines Verlustes, nicht das fröhliche der Hoffnung einflößen." (747.) Dies tue der Dichter der neuen Idylle! „Er führe uns nicht rückwärts in unsre Kindheit, um uns mit 
den kostbarsten Erwerbungen des Verstandes eine Ruhe erkaufen zu lassen, die nicht länger dauern kann als der Schlaf unsrer Geisteskräfte; sondern führe uns vorwärts zu unsrer Mündigkeit, um uns die höhere Harmonie zu empfinden zu geben, die den Kämpfer belohnet, die den Überwinder beglückt. Er mache sich die Aufgabe einer Idylle, welche jene Hirtenunschuld auch in Subjekten der Kultur und unter allen Bedingungen des rüstigsten feurigsten Lebens, des ausgebreitetsten Denkens, der raffiniertesten Kunst, der höchsten gesellschaftlichen Verfeinerung ausführt, welche, mit einem Wort, den Menschen, der nun einmal nicht mehr nach Arkadien zurückkann, bis nach Elysium führt.“ (750.) Der Begriff dieser Idylle ist also „kein andrer als das Ideal der Schönheit, auf das wirkliche Leben angewendet. “" (751.)

Ins wirkliche Leben einzugreifen und es zu verwandeln ist aber, Schillers Theorie zufolge, Aufgabe aller Kunst.

\section{III.}

"Naiv" und „Sentimentalisch“, für Schiller Kategorien des geschichtlichen Verstehens, Kategorien zur Rechtfertigung der eigenen Dichtung und Ausdruck seines Mutes, den Blick vorwärts und nicht zurück zu wenden, verflüchtigen sich bei Schelling zu bloß formellen Momenten: „Die Gegensätze aber, die in Ansehung der Kunst durch ihre Zeitabhängigkeit gesetzt sind, sind, wie die Zeit selbst, notwendig unwesentliche und bloß formelle Gegensätze, ganz verschieden also von den realen im Wesen oder der Idee der Kunst selbst gegründeten. Dieser allgemeine und durch alle Zweige der Kunst hindurchgehende formelle Gegensatz ist der der antiken und modernen Kunst. - Es wäre ein wesentlicher Mangel der Construktion, wenn wir die Rücksicht darauf bei jeder einzelnen Form der Kunst vernachlässigen wollten. Da aber dieser Gegensatz als ein bloß formeller angesehen wird, so die Construktion eben in der Negation oder Aufhebung bestehend." (Philosophie der Kunst, V, $372^{15}$.) „Die Poesie in ihrer Absolutheit ist an sich weder naiv noch sentimental. - Der ganze Gegensatz ist also selbst ein subjektiver, ein bloßer Erscheinungsgegensatz." (V, 473.)

Das absolute Kunstwerk steht vielleicht noch aus: „Denn wenn die ästhetische Produktion von Freiheit ausgeht, und wenn eben für die Freiheit jener Gegensatz der bewußten und der unbewußten Tätigkeit ein absoluter ist, so gibt es eigentlich auch nur Ein absolutes Kunstwerk, welches zwar in ganz verschiedenen Exemplaren existiren kann, aber doch nur Eines ist, wenn es gleich in der ursprünglichsten Gestalt noch nicht existiren sollte." (System des transzendentalen Idealismus, III, 627.)

15 Schellings Werke werden zitiert nach der von seinem Sohn, K. F. A. Schelling veranstalteten Ausgabe, Stuttgart und Ausgburg 1856-1861. 
Dieses vollkommene Werk wird jedoch nicht in Verbindung gebracht mit einem künftigen Reich menschlicher Vollendung und Harmonie, das auch Schelling kennt: „In den Ideen der Philosophie endet die Geschichte mit dem Vernunftreich, d. h. mit dem goldenen Zeitalter des Rechts, wenn alle Willkür von der Erde verschwunden ist, und der Mensch durch Freiheit an denselben Punkt zurückgekehrt sein wird, auf welchen ihn ursprünglich die Natur gestellt hatte, und den er verließ, als die Geschichte begann ..." (III, 589.)

Das Kunstwerk eröffnet, Schellings ontologischer Konzeption gemäß, jenen anderen Bereich der Utopie: Versöhnung von Natur und Geist: "Jedes herrliche Gemälde entsteht dadurch gleichsam, daß die unsichtbare Scheidewand aufgehoben wird, welche die wirkliche und idealische Welt trennt, und ist nur die Öffnung, durch welche jene Gestalten und Gegenden der Phantasiewelt, welche durch die wirkliche nur unvollkommen hindurchschimmert, völlig hervortreten.“ (III, 628.) „Diese Schönheit, welche aus der vollkommenen Durchdringung sittlicher Güte mit sinnlicher Anmut hervorgeht, ergreift und entzückt uns, wo wir sie finden, mit der Macht eines Wunders. Denn weil sich der Naturgeist sonst überall als von der Seele unabhängig, ja gewissermaßen ihr widerstrebend zeigt, so scheint er hier wie durch eine freiwillige Übereinstimmung und wie durch das innere Feuer göttlicher Liebe mit der Seele zu verschmelzen; den Beschauenden überfällt mit plötzlicher Klarheit die Erinnerung an die ursprüngliche Einheit des Wesens der Natur mit dem Wesen der Seele: die Gewißheit, daß aller Gegensatz nur scheinbar, die Liebe das Band aller Wesen, und reine Güte Grund und Inhalt der ganzen Schöpfung ist." (Über das Verhältnis der bildenden Künste zu der Natur. VII, 315/316.)

*

Geschichte, von Schelling zum unwesentlichen Moment erklärt, hat im Systeme Hegels die Kunst überwunden. Kunst wird als ein Vergangenes gesetzt, denn "uns gilt die Kunst nicht mehr als die höchste Weise, in welcher die Wahrheit sich Existenz verschaff" “. (Vorlesungen über die Ästhetik, ed. Friedrich Bassenge, Berlin 1955, 139.)

Die Dimension der Zukunft scheint abgeschnitten. Nichts steht mehr aus, die Versöhnung ist vollzogen: „die Gegenwart hat ihre Barbarei und unrechtliche Willkür, und die Wahrheit hat ihr Jenseits und ihre zufällige Gewalt abgestreift, so daß die wahrhafte Versöhnung objektiv geworden, welche den Staat zum Bilde und zur Wirklichkeit der Vernunft entfaltet ..." (Grundlinien der Philosophie des Rechts, ed. Johannes Hoffmeister, Hamburg 1955, 297, $\$ 360$.

Das absolute Bewußtsein, das alle Wirklichkeit in sich aufgenommen hat, ist schlechthin Gegenwart; Gegenwart aber, die trächtig ist von Erinnerung, 
ja die nichts anderes ist als Er-Innerung: „Der Geist hat alle Stufen der Vergangenheit noch an ihm, und das Leben des Geistes in der Geschichte ist, ein Kreislauf von verschiedenen Stufen zu sein, die teils gegenwärtig, teils in vergangener Gestaltung erschienen sind. Indem wir es mit der Idee des Geistes zu tun haben und in der Weltgeschichte alles nur als seine Erscheinung betrachten, so beschäftigen wir uns, wenn wir Vergangenheit, wie groß sie auch immer sei, durchlaufen, nur mit Gegenwärtigem. Die Philosophie hat es mit dem Gegenwärtigen, Wirklichen zu tun. Die Momente, die der Geist hinter sich zu haben scheint, hat er auch in seiner gegenwärtigen Tiefe ..." (Die Vernunft in der Geschichte, ed. Hoffmeister, Hamburg 1955, 183.) „... Die Er-Innerung hat (die Erfahrung der frühern Geister) aufbewahrt und ist das Innre und die in der Tat höhere Form der Substanz ... Das Ziel, das absolute Wissen, oder der sich als Geist wissende Geist hat zu seinem Wege die Erinnerung der Geister... Ihre Aufbewahrung nach der Seite ihres freien in der Form der Zufälligkeit erscheinenden Daseins, ist die Geschichte, nach der Seite ihrer begriffnen Organisation aber die Wissenschaft des erscheinenden Wissens; beide zusammen, die begriffne Geschichte, bilden die Erinnerung und die Schädelstätte des absoluten Geistes, die Wirklichkeit, Wahrheit und Gewißheit seines Throns, ohne den er das leblose Einsame wäre; nur aus dem Kelche dieses Geisterreiches schäumt ihm seine Unendlichkeit."

(Phänomenologie des Geistes, Schluß; ed. Hoffmeister, Leipzig 1949, 564.)

Von solcher Glorifizierung der "begriffnen Geschichte" durch das absolute Bewußtsein leben seitdem weithin die historischen Wissenschaften ${ }^{16}$. Die Überzeugung von der im Gegenwärtigen vollzogenen Versöhnung schwindet bald. Und an die Stelle der Erwartung eines künftigen Reiches der Vollendung als Aufgabe des menschlichen Geistes und Willens tritt nun die Erinnerung an eine schon vollbrachte, vergangene Einstimmigkeit und Erfüllung des geschichtlichen Daseins als eines Faktums ${ }^{17}$.

16 „In der Tat gilt fast ausnahmslos, daß wir, wo immer wir einem Wort der deutschen historischen Geisteswissenschaft tiefer nachdenken, auf Hegel stoßen ...“ (Helmut Kuhn: Wesen und Wirken des Kunstwerks. München 1960, 17.)

${ }^{17} \mathrm{Vgl}$. hierzu Hermann Cohen: Kants Begründung der Aesthetik. Berlin 1889, 338/339: „Geschichte wird die Macht, von welcher aller Werte abgeleitet werden. Wissenschaft und Moral werden in der Geschichte, in der Religion der sogenannten Thatsachen, oder aber auch der Mythen gegründet. Denn besser, man macht die Geschichte und die Erfahrung zur Schatzkammer des Heiligen, als daß man das zweischneidige Schwert des menschlichen Bewußtseins zum Rüstzeug der Kultur beglaubigt. - Nun haben aber Thatsachen nicht die Gewißheit, mit der allein der Moral geholfen werden kann; und Erfahrung und Geschichte ersetzen nicht die Rechtfertigung des Bewußtseins. Der Romantik gebricht es im letzten Grunde an nichts Anderem als am Glauben; an dem Glauben nämlich in die Un- 
Diese Wendung des Blicks von der Zukunft weg auf die Vergangenheit und damit die Umformung einer Forderung, eines Sollens, zur Kontemplation eines "Gegebenen " ist zu fassen an einem Zentralbegriff ${ }^{18}$ der Kunstgeschichtswissenschaft, dem Stilbegriff. Als zugleich ästhetische und historische Kategorie befaßt er in sich die formalen Bestimmungen, die Kant und Schiller jenem künftigen Reiche zugesprochen hatten: Einheit, Einstimmigkeit, Totalität, und trägt anfänglich auch dessen Wertcharakter von Vollendung und Vollkommenheit.

Goethes Terminologie macht den Wertgehalt des Stilbegriffs sichtbar: „Es ist uns ... angelegen, das Wort Stil in den höchsten Ehren zu halten, damit uns ein Ausdruck übrig bleibe, um den höchsten Grad zu bezeichnen, welchen die Kunst je erreicht hat und je erreichen kann." (Einfache Nachahmung der Natur, Manier, Stil. Hamburger Ausgabe, XII, Hamburg 1953, 34.)

Winckelmann überträgt den Begriff unter Wahrung seines Wertcharakters auf die Geschichte. Der „wahre gute Geschmack“ des Griechentums faßt den Inbegriff der Schönheit in sich. Deshalb kann „Stil“ nur den Griechen zugesprochen werden. Der Begriff eines "römischen Stils“ ist für Winckelmann „irrig": „Die römischen Künstler sind als Nachahmer der Griechen anzusehen und haben also keine besondere Schule und keinen eigenen Stil bilden können." (Geschichte der Kunst des Altertums, VIII, ed. Eiselein, 276, 265.)

Winckelmanns historische Fixierung des Stils macht seine eigene Forderung nach Wiederherstellung der griechischen Kunst zunichte. „An die Stelle seines optimistischen Erneuerungsglaubens tritt die Resignation; Winckelmann zieht sich aus der künstler-pädagogischen Position zurück und sucht in der Sphäre der reinen Erkenntnis Ersatz für den unwiederbringlich verlorenen Besitz. " ${ }^{19}$

Die komplexe Geschichte des Stilbegriffs in ihrer Verflechtung mit der Ausbildung des historischen Bewußtseins kann hier nicht dargestellt werden. Aufschlußreich ist, daß im Grundbuch der wissenschaftlichen Stilgeschichte die Kon-

bezwinglichkeit und unergründliche Tiefe der rastlos ringenden Uberzeugung; daher fehlt ihr die unendliche Fernsicht der Hoffnung auf das Ende der Tage, auf eine dereinstige irdische Darstellung der Menschheit. Woran es der Romantik aber keineswegs fehlt, das ist das lebendige Gefühl für die Mittel, jenen Traumwunsch der Menschheit als erledigt zu erklären. Wie im goldenen Zeitalter alle Ideale realisiert gewesen seien, so ist in Religion, Politik und Kunst der Anfang der Geschichte stets das Ziel und die Losung. Der Mythos vom Paradiese ist die Signatur der Romantik. Wenn man nur auf das von der Geschichte bereits und zwar vorlängst Geleistete zurückgehen wollte, so könnte man alles ferneren Ringens nach neuen Idealen überhoben sein. So wird die Geschichte der Erfahrung zum prähistorischen Mythos."

18 "Mit dem Begriff des Stils ist nämlich zum erstenmal ein wissenschaftliches Prinzip aufgestellt worden, das als die Grundlage einer wirklich kunstgeschichtlichen Methode betrachtet werden kann." (Friedrich Kreis: Der kunstgeschichtliche Gegenstand. Ein Beitrag zur Deutung des Stilbegriffs. Stuttgart 1928, 17.)

19 Ingrid Kreuzer: Studien zu Winckelmanns Ästhetik. Normativität und historisches Bewußtsein. Berlin 1959, 100. 
zeption des Stilbegriffs, reduziert auf "Einheit“, zusammengeht mit seiner polemischen Abhebung von einer „zerrissenen“ Gegenwart: „Nichts bezeichnet eindrücklicher den Gegensatz zwischen alter Kunst und der Kunst von heute als die Einheitlichkeit der Sehform dort und die Vielfältigkeit der Sehformen hier. In einer Weise, die einzig ist in der bisherigen Kunstgeschichte, scheint das Widersprechendste sich miteinander vertragen zu können ... Was bedeuten daneben einzelne auseinandergehende Richtungen der Vergangenheit! ... Es ist eine schöne Aufgabe der wissenschaftlichen Kunstgeschichte, wenigstens den Begriff eines derartig einheitlichen Sehens lebendig zu erhalten, das verwirrende Durcheinander zu überwinden und das Auge in ein festes und klares Verhältnis zur Sichtbarkeit zu bringen." (Wölfflin ${ }^{20}$.) Nach Sedlmayr hat die Stilgeschichte an der Erfassung der Kunst des 19. Jahrhunderts ihre Grenze: „Die Stilgeschichte konnte zur Kennzeichnung einer Epoche nur jene künstlerischen Erscheinungen zusammenfassen, die noch unter einen einheitlichen Stilbegriff zu subsumieren sind ... Aber sie war von sich aus unfähig, zu erfassen, daß unter Umständen auch künstlerische Erscheinungen ganz verschiedenen, ja widersprechenden Stils doch aus ein und derselben geistigen Wurzel erwachsen können. ${ }^{\text {"21 }}$ Diese Erkenntnis leiste der Begriff der "Struktur“. Daß das 19. und 20. Jahrhundert zerrissen und chaotisch seien, das Prinzip ihrer Einheit die "Desintegration" sei, gilt weiterhin als ausgemacht.

Hier spiegelt der Stilbegriff die unabdingbare Forderung der Vernunft nach Einheit und Totalität in die Vergangenheit zurück. Diese Geschichtsbetrachtung ist „ästhetisch“22, weil sie Einheit und Totalität als Gegebenheiten vorzufinden glaubt. Nur im Kunstwerk aber sind Einheit und Totalität, das heißt: Weltsetzung vollzogen und damit anschaubar, - nicht in der Geschichte, auch nicht in der Kunstgeschichte.

"Welt ist eine Denknotwendigkeit, die zugleich das bloße Denken übersteigt. Sie erscheint qua Denken als Forderung, da sonst der Mensch schließlich

${ }^{20}$ Heinrich Wölfflin: Kunstgeschichtliche Grundbegriffe. Das Problem der Stilentwicklung in der neueren Kunst. München 1915, IX/X.

21 Hans Sedlmayr: Die Grenzen der Stilgeschichte und die Kunst des 19. Jahrhunderts. Wiederabgedruckt in: Der Tod des Lichtes. Salzburg 1964, 101.

22 "In der Konzeption der Stilgeschichte ist eben schon von Geburt an die Tendenz zu einer der historischen Wirklichkeit kaum einmal entsprechenden Harmonisierung dadurch gegeben, daß der Stilbegriff ursprünglich von der Geschlossenheit eines einzelnen Kunstwerks auf die Geschlossenheit einer künstlerischen Epoche übertragen worden ist..." (Sedlmayr, 102). Aber auch die Kategorie der "Struktur“ wurde vom Kunstwerk auf die Geschichte übertragen: „Es kommt jetzt darauf an, mit streng wissenschaftlichen Verfahren an einer Epoche aus wenigen Voraussetzungen möglichst vieles, aus diesen Voraussetzungen mit innerer Folgerichtigkeit Folgendes ähnlich zu verstehen, wie man vorher an einem Kunstwerk aus möglichst wenig Zentralem möglichst viel Peripheres verstanden hat.“ (Sedlmayr: Kunst und Wahrheit. Zur Theorie und Methode der Kunstgeschichte. Hamburg 1958, 198.) 
einer letzten Sicherheit im Dasein und im Handeln entbehren müßte... Welt ist eine Notwendigkeit des Glaubens... Welt ist drittens - in der dritten verstehenden Verhaltensweise des Geistes zur Wirklichkeit: in der Kunst nicht als Forderung und nicht als Hoffnung - sondern als Verwirklichung erwiesen. Damit ist der geistige Ort der Kunst bestimmt ... “" (Badt ${ }^{23}$.)

Für das geschichtliche Leben sind Einheit und Totalität unabgeltbare Forderungen an die theoretische und praktische Vernunf, Forderungen, die zur Verwirklichung aufrufen, Forderungen an freie, mündige Menschen, niemals Gegenstände der Betrachtung und Beschreibung, niemals Erinnerungen.

Daß aber auch das Kunstwerk ins Leben, in die Zukunft wirken muß, nicht Repräsentant vergangener Stile ist, das können Kant und Schiller uns sagen.

"Nicht genug also, daß alle Aufklärung des Verstandes nur insoferne Achtung verdient, als sie auf den Charakter zurückfließt; sie geht auch gewissermaßen von dem Charakter aus, weil der Weg zu dem Kopf durch das Herz muß geöffnet werden. Ausbildung des Empfindungsvermögens ist also das dringendere Bedürfnis der Zeit, nicht bloß weil sie ein Mittel wird, die verbesserte Einsicht für das Leben wirksam zu machen, sondern selbst darum, weil sie zu Verbesserung der Einsicht erweckt.“ (Über die ästhetische Erziehung des Menschen, Achter Brief, 592.)

${ }^{23}$ Kurt Badt: Die Idee der Welt und das Selbst als fundamentale Wesenheiten bildender Kunst. In: Festschrift für Walter Friedlaender. 1933. 17/18. Maschinenschriftliches Exemplar im Warburg Institute, London. 